\title{
Fractional order proportional-integral controller applied to a back-to-back converters
}

\author{
Manuel A. Duarte-Mermoud ${ }^{1,2,{ }^{*}}$, Juan-Carlos Travieso-Torres ${ }^{3}$, and Tedy A. Crespo-Herrera ${ }^{1,2}$ \\ ${ }^{1}$ Electrical Engineering Department, Universidad de Chile, Av. Tupper 2007, Casilla 412-3, Santiago, Chile. \\ ${ }^{2}$ Advanced Mining Technology Center, Universidad de Chile, Av. Tupper 2007, Casilla 412-3, Santiago, Chile. \\ ${ }^{3}$ Depto. de Tecnologías Industriales, Facultad Tecnológica, Universidad de Santiago de Chile, Av. El Belloto 3735, Santiago, Chile.
}

\begin{abstract}
The use of fractional order proportional-integral (FOPI) controllers, applied to back-to-back (BTB) power converters is proposed and studied in this paper. A comparative study with the integer order proportional-integral (PI) controllers, traditionally used for this purposes, is performed and the result are discussed. The BTB power converter is connected between a permanent magnet synchronous generator (PMSG) -which is moved by a turbine placed in line with a mineral pipeline for energy recovery purposesand the electrical network, to inject the recovered energy through a voltage control into the electrical network. Simulations under normal conditions (constant torque) as well as under variable torque variations are studied, representing different possible operating scenarios for the generator.
\end{abstract}

\section{Introduction}

The high demand for electrical energy and the environmental constraints have stimulated the development of alternative sources of energy, clean and sustainable [1].

It is possible to recover energy from pipelines used in industrial processes such as mining pipelines, transporting some fluids by gravity or under pressure for long distances and covering height difference of about 3000 meters [2]. A particular case is the pipeline Los Bronces - Las Tórtolas located in Chile, where studies indicate that there exists a potential energy between $22.03 \mathrm{Kw}$ and $34.35 \mathrm{Kw}$.

On the other hand, during the last few decades fractional calculus and fractional control have become very active areas of research in several disciplines like the ones mentioned in [3-6].

The main interest of this paper is focused on the control of systems to recover energy from mining pipelines using power converters and fractional order controllers, instead of the classic PID controllers. Although there exist different types of fractional order controllers (FOC) we will focus on the use of fractional order proportional integral (FOPI) controllers

FOC have also been studied in the context of wind energy systems $[1,7]$ where the wind velocity is much more variable than the flux inside a pipeline. In [7] it is analyzed the case of a two mass rotor and a permanent magnet synchronous generator (PMSG) using a three phase matrix converter AC-AC and a multilevel converter AC-DC-AC, controlled by fractional controllers. In [8] a simulated study in the time domain is performed on a similar system as in [7] using a multilevel converter. An offshore wind turbine system is analyzed in [9] using a PMSG, a multilevel converter of three and four levels with neutral point clamped (NPC), reporting in all cases a reduction in the harmonic distortion as compared with integer order controllers.

In [10] the rectifier stage is done by a static non controlled rectifier bridge, the DC control is performed through a Boost (DC-DC) actuated by a FOPI controller and uses a three level NPC in the inversion (DC-AC) stage. In [11] a DC-DC converter is controlled by FOPI and FOPID within a sliding mode control, whereas in [12] they compare a FOPI with a PI applied to a DC-DC converter. The results obtained in [7-12] are similar to those obtained in $[25,26]$ with more complex controllers.

In this paper, we propose and analyse a back-to-back (BTB) power converter connected between a PMSG and an electrical network, employing FOPI controllers in different places of the control scheme, instead of the commonly used PI controllers. The results are compared with the case of using classic PI controller. In Section II the system to be studied is described, whereas in Section III the control system is explained in detail. Section IV presents the results of simulating the proposed strategy under different operating conditions. Finally, in Section $\mathrm{V}$ some conclusions are drawn.

\section{System description}

Figure 1 shows the general block diagram of the energy recovery system and its control scheme. It is composed by a PMSG, two converters in a back-to back (BTB) configuration commuted with space vector modulation (SVM) technique and connected to the electrical network (grid).

Corresponding author: mduartem@ing.uchile.cl 
The machine side converter (MSC) is controlled by the MSC control system and the grid side converter (GSC) is controlled by the GSC control system.

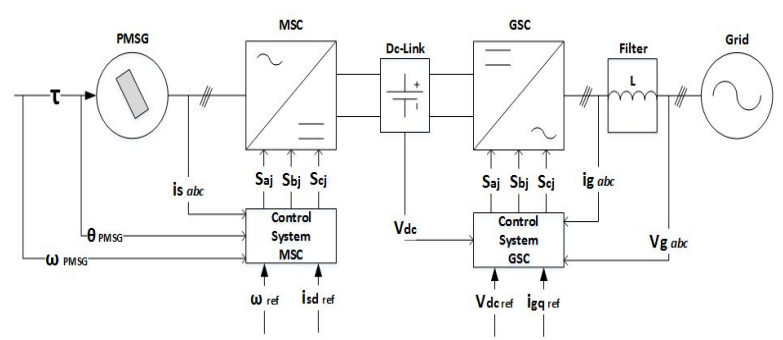

Fig. 1. Energy recovery system.

\subsection{PMSG}

The PMSG is characterized since it does not have an external excitation circuit but it has permanent magnets in the rotor. This generator has important advantages which includes the elimination of slip rings and the losses in the field coil [13]. The PMSG is of a smaller size and possess a much smaller moment of inertia.

The model of the PMSG is usually used in terms of a reference framework rotating at synchronous speed denoted as $\mathrm{d}-\mathrm{q}$ axes, obtained from the Park transformation [27] in terms of the direct and quadrature stator voltages $v_{s d}, v_{s q}$ and currents $i_{s d}, i_{s q}$ are related as

$$
\begin{aligned}
& v_{s d}=R_{s} i_{s d}+\frac{d \psi_{d}}{d t}-\omega_{e} \psi_{q} \\
& v_{s q}=R_{s} i_{s q}+\frac{d \psi_{q}}{d t}+\omega_{e} \psi_{d}
\end{aligned}
$$

where the stator fluxes $\psi_{\mathrm{d}}$ and $\psi_{\mathrm{q}}$, are given by

$$
\begin{aligned}
& \psi_{d}=L_{d} i_{s d}+\psi_{m} \\
& \psi_{q}=L_{q} i_{s q}
\end{aligned}
$$

where $R_{s}$ is the stator resistance, $\omega$ is the electrical frequency and $\psi_{m}$ is the magnetizing flux.

The electromagnetic torque is expressed as

$$
T_{e}=\frac{3}{2} p\left[\psi_{m} i_{q}+\left(L_{d}-L_{q}\right) i_{d} i_{q}\right]
$$

and the mechanical rotor speed is obtained as

$$
\dot{\omega}_{m}=\frac{1}{J}\left(T_{e}-T_{m}\right)
$$

where $L_{d}, L_{q}$ are the direct and quadrature inductances, $J$ is the rotor moment of inertia and $T_{m}$ is the mechanical torque on the rotor axis.

\subsection{Power converters}

The two levels three phase power converters are formed by six switches ( 2 per phase) which are considered ideals for this study. The topology of the BTB converter is shown in Figure 2 [14].

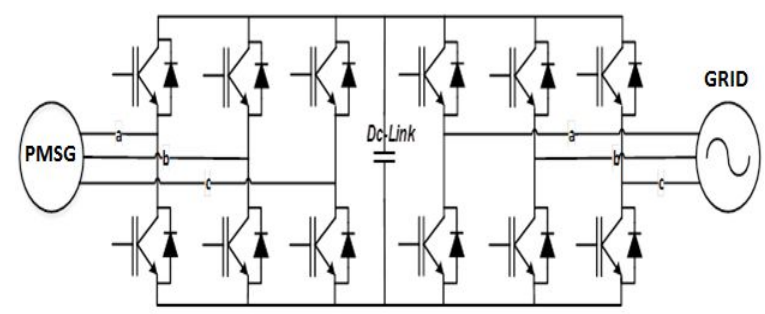

Fig. 2. Topology of the BTB converter.

\subsection{Phase-locked loop}

The phase-locked loop (PLL) is a circuit that synchronizes the output signal with a reference or input signal, in frequency as well as in phase [15]. For controlling the power inverter of the grid side it is necessary to synchronize the converter with the network frequency $\omega_{g}$. Also it is required to determine the phase to compute the $d q$ transformations used in the controllers [16]. The block diagram of the PLL is shown in Figure 3.

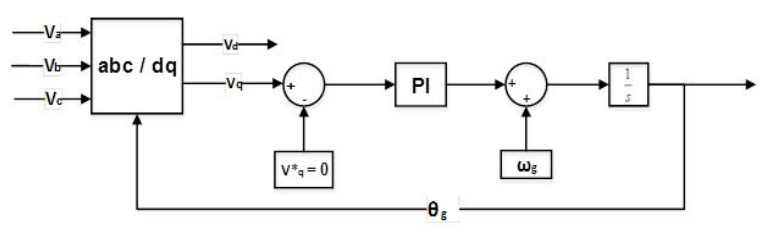

Fig. 3. Block diagram of the PLL.

In order to tune the PI controller of the PLL, the methodology described in [28,29] is used, where a second order transfer function is assumed for this system.

\section{Proposed control system}

In what follows the proposed control system used for this study is described in detail.

\subsection{Fractional order PI controller}

The term fractional control refers to the extension of classical control design that uses integer order operators (integrals and derivatives) to the case when fractional order operators are introduced in the controller design [17]. The definition of the fractional order integral according to Riemmann-Liouville is given by [18]

$$
I^{\lambda} f(t)=\frac{1}{\Gamma(\lambda)} \int_{0}^{t}(t-\tau)^{\lambda-1} f(\tau) d \tau
$$

with $t>0 ; \lambda \in \mathfrak{R}^{+}$, and $\Gamma(\lambda)$ is the Gamma function. Assuming zero initial conditions (IC), in the frequency domain (Laplace Transform) this fractional integral is expressed as 


$$
\mathcal{L}\left\{I^{\lambda} f(t)\right\}=\frac{1}{s^{\lambda}} F(s)
$$

There exists several definitions for the fractional order derivative (FOD), however the most used in engineering applications is the Caputo derivative given by [19], [20]

$$
{ }^{C} D_{t}^{\mu} f(t)=\frac{1}{\Gamma(1-\delta)} \int_{0}^{t} \frac{f^{m+1}(\tau)}{(t-\tau)^{\delta}} d \tau
$$

where $\mu=m+\delta ; m \in \mathfrak{R}, 0 \leq \delta \leq 1$, whose Laplace transform (assuming zero IC) us given by

$$
\mathcal{L}\left\{D^{\mu} f(t)\right\}=s^{\mu} F(s)
$$

The generalization of the classic PID controller to its fractional form FOPID was introduced in [20]. In particular, the FOPI controller in the frequency domain will be used as follows

$$
F O P I=k_{p}+\frac{k_{i}}{s^{\lambda}}
$$

with $\lambda \in \mathfrak{R}^{+}$. From (11) it is possible to generate different kinds of PI controllers by assigning particular values to. $\lambda \in \mathfrak{R}^{+}$. For instance if $\lambda=1$ it becomes the standard PI controller; if $\lambda=0$ the standard P controller is generated. For real values of $\lambda \in \mathfrak{R}^{+}$infinite FOPI controllers are obtained [10].

\subsection{Converter fractional order control scheme}

The most commonly used control scheme in power converters is the vector control (VC) [21]. The block diagram of the machine side controller (MSC) is shown in Figure 4, where the components Compd, Comp $q$ are used to decouple torque and flux and they are given by [11]

$$
\begin{aligned}
& \operatorname{Comp}_{d}=\omega i_{s q} L_{q} \\
& \operatorname{Comp}_{q}=\omega i_{s d} L_{d}+\psi_{m}
\end{aligned}
$$

In Figure 4 the proposed MSC is shown, where the traditionally used PI controllers have been replaced by the FOPI controllers.

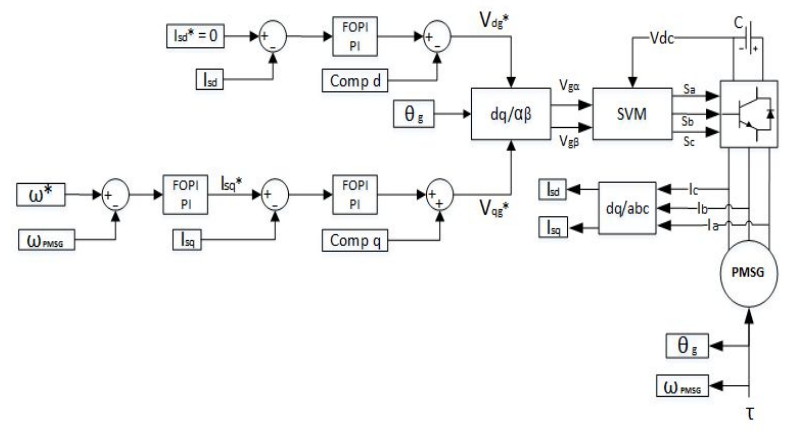

Fig. 4. Proposed MSC.

The proposed block diagram of the grid side controller (GSC) is shown in Figure 5. The control strategy is based on the coordinate transformation $a b c$ $d q$. In [12] it is stated that this strategy guarantees a rapid transient response and a high static performance. Note that again we have used FOPI instead of PI controllers.

The control loop scheme of Figure 6 is used to design the current controllers in the MSC. This loop contains a FOPI, a first order transfer function representing the power converter with a time constant depending on the commutation frequency $\left(T_{s w}=1 / f_{s w}\right)$, and the block is determined by the voltage equations in dq axes after the coupling terms are eliminated.

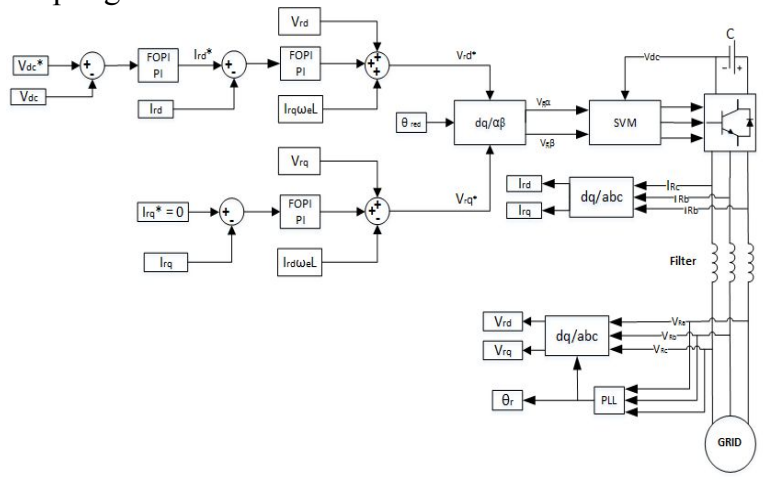

Fig. 5. Proposed GSC.

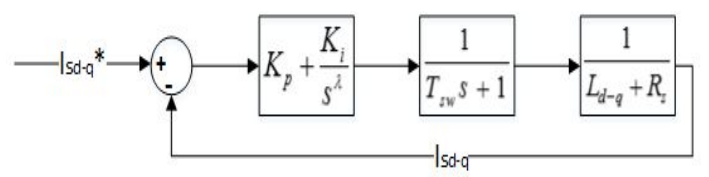

Fig. 6. Scheme of current control loop for MSC.

The control loop scheme of Figure 7, employed in the design of the speed controllers of the MSC, contains the FOPI controller, a delay produced by the current controller, the electrical torque constant given by (5) (neglecting the mechanical torque since it is considered as a perturbation) and the equation (6) to obtain the speed of the electrical machine, with $\tau_{i s q}=R_{s} / K_{i-i s q}$ where $K_{i-i s q}$ is the overall gain of the current controller.

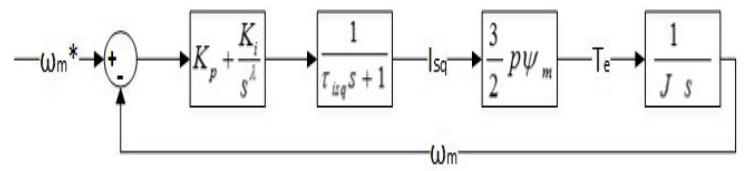

Fig. 7. Scheme of MSC speed control loop.

The controller parameters shown un Table 1, were obtained for each controller (current and speed of MSC) from an optimization problem using the Particle Swarm Optimization (PSO) method. It was solved by sequential quadratic programming (SQP) implemented using the toolbox FOMCON [23] of Matlab/Simulink. The optimization function used was the integral of the square error (ISE).

Table 1. Parameter values for MSC controllers.

\begin{tabular}{|c|c|c|c|}
\cline { 2 - 4 } \multicolumn{1}{c|}{} & $\mathbf{K}_{\mathbf{p}}$ & $\mathbf{K}_{\mathbf{i}}$ & $\boldsymbol{\lambda}$ \\
\hline $\boldsymbol{I s}_{\boldsymbol{d}-\boldsymbol{q}}$ & 0.8679 & 1.2936 & 0.5105 \\
\hline $\boldsymbol{\omega}_{\boldsymbol{m}}$ & 832.01 & 50428 & 0.9 \\
\hline
\end{tabular}


The control loop for currents $d q$ of the GSC is show in Figure 8. This is similar to the MSC current control scheme but the currents are given by the firs order transfer function of filter $L$ located at the output.

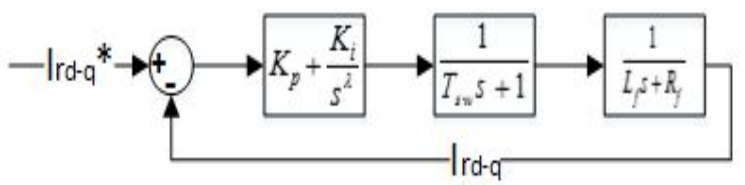

Fig. 8. Scheme of current control loop for GSC.

Figure 9 shows the control loop for the DC-Link to maintain the voltage level in the GSC. It is composed by its FOPI controller and the delay generated by the current controller given by [22]. The voltage level is obtained through the transfer function of the capacitor of the DC-Link.

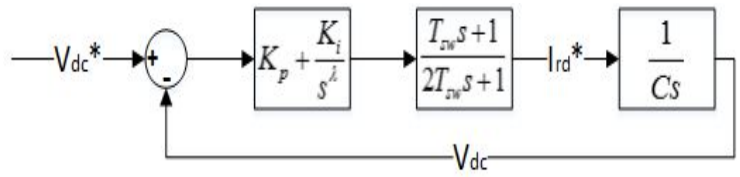

Fig. 9. Scheme of current control loop for GSC.

The parameters obtained via PSO adjustment procedure for each controller (current and DC-Link of GSC) are shown in Table 2.

Table 2. Parameter values for GSC controllers

\begin{tabular}{|c|c|c|c|}
\cline { 2 - 4 } \multicolumn{1}{c|}{} & $\mathbf{K}_{\mathbf{p}}$ & $\mathbf{K}_{\mathbf{i}}$ & $\boldsymbol{\lambda}$ \\
\hline $\mathbf{I r}_{d q}$ & 1.26 & 1.048 & 0.39 \\
\hline DC-Link & 50 & 6140.7 & 0.89 \\
\hline
\end{tabular}

\section{Simulation results}

The system described in Sections 2 and 3 is simulated under different operating conditions. First, a constant torque corresponding to the nominal value is applied on the system (see Figure 10(a)). Next, the system us subjected to a variable torque having fluctuations of $15 \%$ of the nominal value (see Figure 10(b)), which represent typical variations of the flux and pressure of the pipeline.
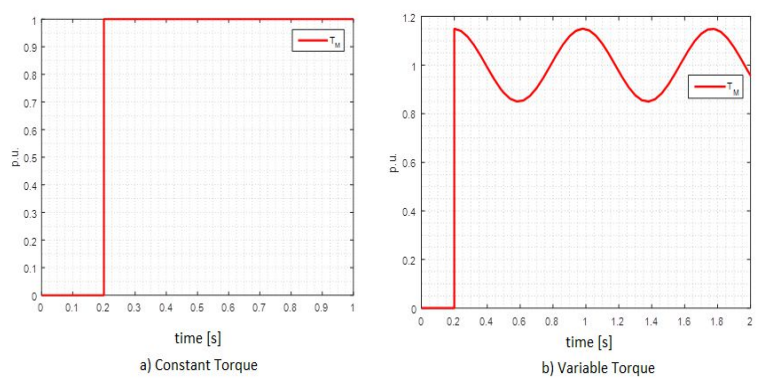

Fig. 10. Input torques to the system.

For comparison purposes the control schemes shown in Figure 4 and Figure 5 were also simulated using classic PI controllers. The PI settings were found using the Root Locus method (RLM) available in Matlab imposing, for the closed-loop response under a step reference change, with a settling time of $0.25 \mathrm{~s}$ and a $1 \%$ of maximum overshoot (MO) obtaining the parameters of Table 3 .

Table 3. PI Parameters values for MSC and GSC.

\begin{tabular}{|c|c|c|}
\cline { 2 - 3 } \multicolumn{1}{c|}{} & $\mathbf{K}_{\mathbf{p}}$ & $\mathbf{K}_{\mathbf{i}}$ \\
\hline $\boldsymbol{I s}_{\boldsymbol{d}-\boldsymbol{q}}$ & 0.577 & 0.274 \\
\hline $\boldsymbol{\omega}_{\boldsymbol{m}}$ & 861.015 & 57401 \\
\hline $\boldsymbol{I r}_{\boldsymbol{d - q}}$ & 1.29 & 26.877 \\
\hline $\boldsymbol{D} \boldsymbol{C}$ - Link & 29.7 & 5941.3 \\
\hline
\end{tabular}

Figure 11 shows the controllers response for the speed. It is observed that the overshoot obtained in both cases (constant and variable torque) is smaller when FOPI are used.

Figure 12 presents the responses of the DC-Link voltage under both type of torques applied, using both types of controllers. Again it is observed that the overshoot obtained with the FOPI is smaller as compared with that obtained with the PI.

The percentage of error (with respect to the set point) for both type of controllers, for the speed as well as for the DC-Link voltage, are shown in Figures 13 and 14. For both controllers the steady state error converges to zero, however the variations produced by the PI controllers are larger than those obtained for the FOPI.

In order to quantify the differences in the performance of both controllers two performance indexes where evaluated; the integral of the absolute value of the error (IAE) and the integral of the square error (ISE). The results are tabulated un Tables 4 and 5, respectively. In the case of the FOPI when the torque is constant the ISE indexes for speed and DC-Link voltage are $85.76 \%$ and $67.83 \%$ smaller than the respective values obtained with the PI controllers. In the case of a variable torque the IAE of the FOPI for the speed loop is $91 \%$ smaller than the PI controller, whereas the ISE of the FOPI for DC-Link voltage loop is $66.5 \%$ smaller that the corresponding PI controller.

\section{Conclusions}

A fractional order control scheme has been proposed for controlling a back-to-back power converter connected between a permanent magnet synchronous generator and an electrical network. The PMSG is driven by a mechanical torque obtained from a mineral pipeline of variable flux and pressure. A comparison between integer order proportional-integral controllers with the corresponding fractional order proportional-integral controllers is studied and discussed.

Due to the additional number of degrees of freedom (DOF) in the case of the FOPI controller (three DOF), as compared with the PI controller (two DOF), the former presents a better behavior measured through the IAE and ISE performance indexes. 


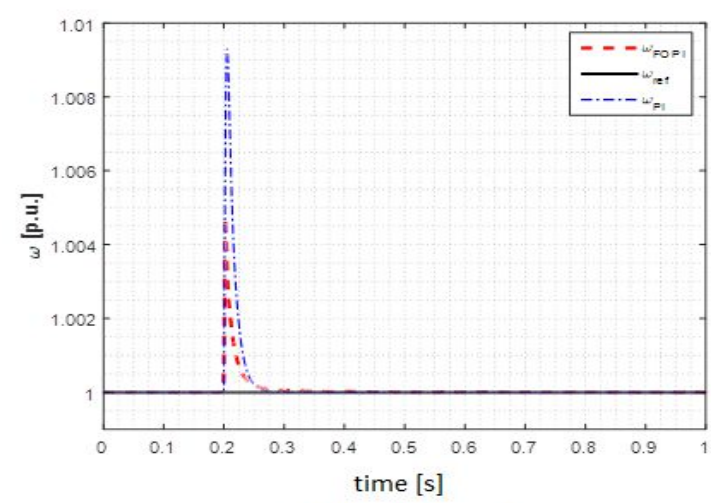

a) Constant Torque

Fig. 11. Speed controllers' response.

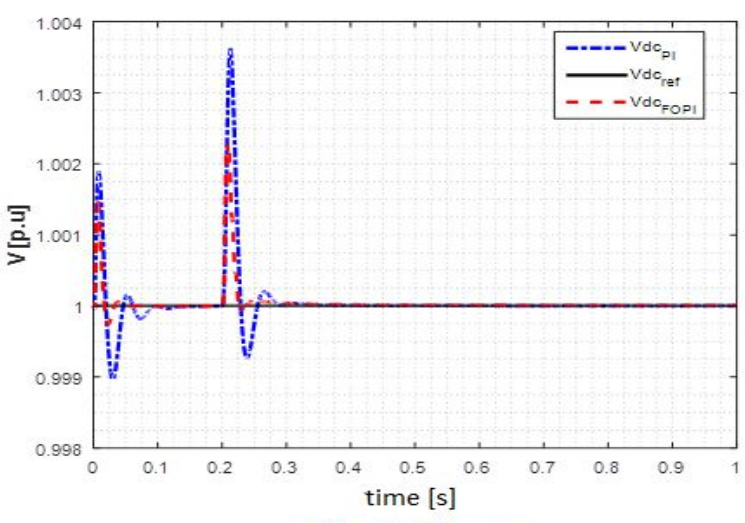

a) Constant Torque

Fig. 12. Controllers' response for DC-Link.

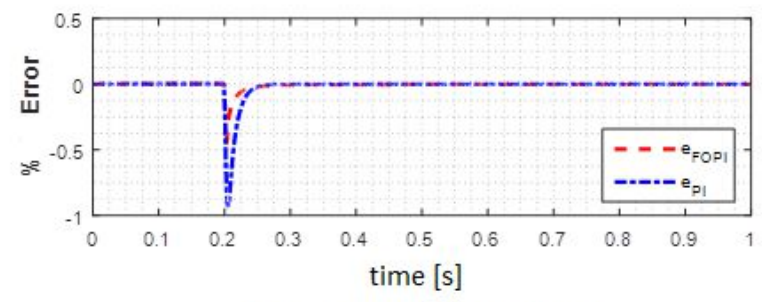

a) Speed (at constant torque)

Fig. 13. Controller's error percentage for constant Torque.

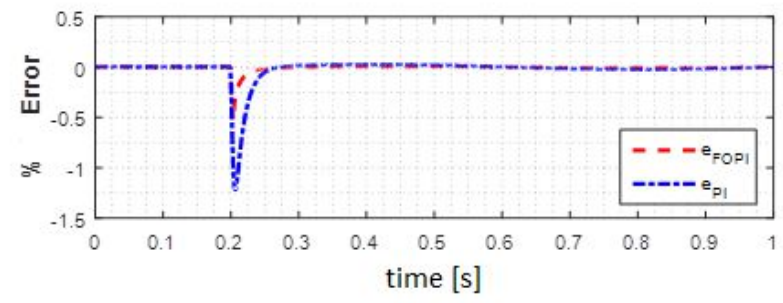

a) Speed (at variable torque)

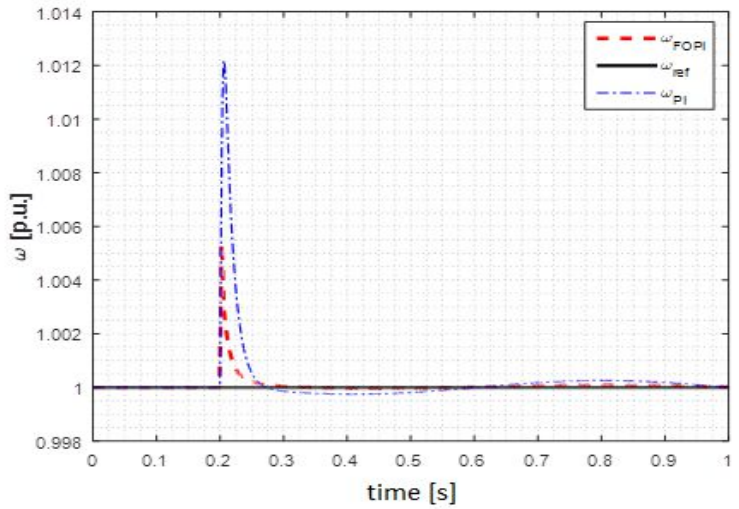

b) Variable Torque

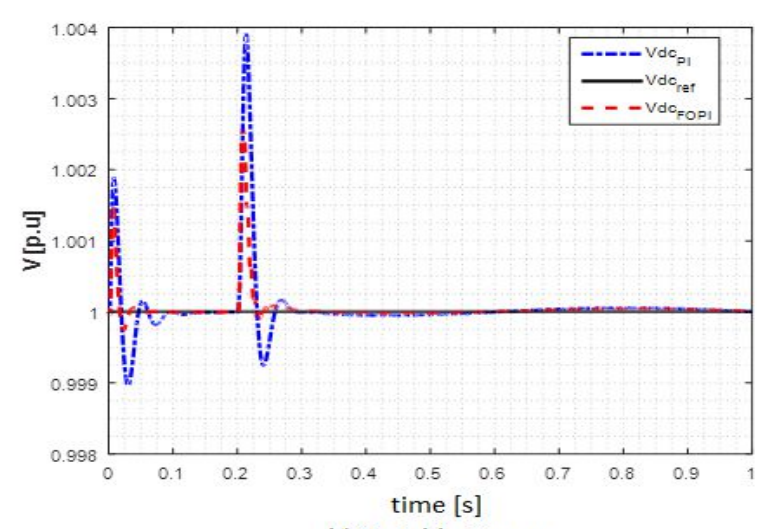

b) Variable Torque

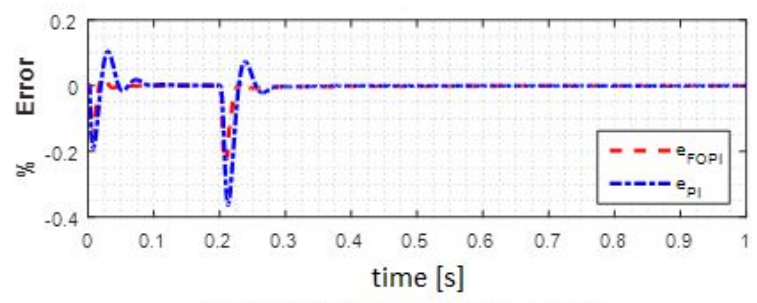

b) DC-Link (at constant torque)

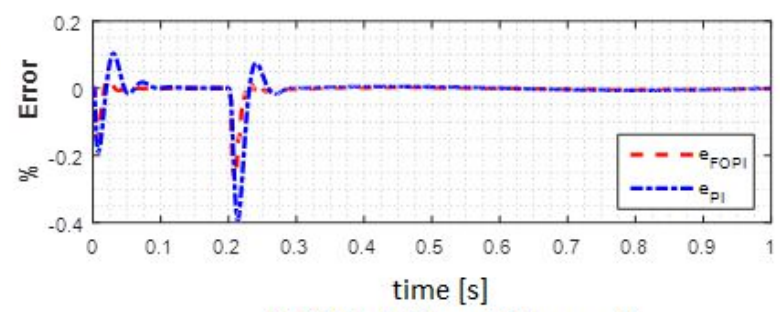

b) DC-Link (at variable speed)

Fig. 14. Controllers' error percentage for variable Torque. 
The resulting indexes are tabulated in Tables 3 and 4, respectively From there we can conclude that when a step torque of magnitude 1 [p.u.] is applied from zero IC the ISE indexes for the variables speed and DC-Link voltage are $85.8 \%$ and $67.8 \%$ smaller than those obtained when using PI controllers.

For the case when a sinusoidal torque is applied to the PMSG, the IAE associated to the FOPI controller for the speed loop is $91.0 \%$ smaller than the obtained for the PI controller. Furthermore, the ISE of the FOPI controller for DC-Link voltage loop is $66.5 \%$ smaller that the corresponding to the PI controller.

Table 4. Performance indexes of the controllers under constant torque.

\begin{tabular}{|c|c|c|c|c|}
\cline { 2 - 5 } \multicolumn{1}{c|}{} & \multicolumn{2}{c|}{ PI } & \multicolumn{2}{c|}{ FOPI } \\
\cline { 2 - 5 } \multicolumn{1}{c|}{} & $\boldsymbol{\omega}$ & DC-Link & $\boldsymbol{\omega}$ & DC-Link \\
\hline IAE & 0.04741 & 0.4767 & 0.024 & 0.2378 \\
\hline ISE & 0.08409 & 3.405 & 0.01198 & 1.096 \\
\hline
\end{tabular}

Table 5. Performance indexes of the controllers under variable torque.

\begin{tabular}{|c|c|c|c|c|}
\cline { 2 - 5 } \multicolumn{1}{c|}{} & \multicolumn{2}{c|}{ PI } & \multicolumn{2}{c|}{ FOPI } \\
\cline { 2 - 5 } \multicolumn{1}{c|}{} & $\boldsymbol{\omega}$ & DC-Link & $\boldsymbol{\omega}$ & DC-Link \\
\hline IAE & 0.1139 & 0.5987 & 0.0305 & 0.2823 \\
\hline ISE & 0.183 & 4.016 & 0.0158 & 1.344 \\
\hline
\end{tabular}

This research has been supported by CONICYT-Chile, through FONDECYT Project 1150488 "Fractional Error Models in Adaptive Control and Applications" and by the Programa de Financiamiento Basal "Centro de Tecnología para la Minería" FB0809. T.A.C.H. thanks the support of CSIRO-Chile to his Master Thesis.

\section{APPENDIX}

Table 5. Parameter values for MSC controllers.

\begin{tabular}{|c|c|}
\hline \multicolumn{2}{|c|}{ Parameters of the PMSG } \\
\hline Parameter & Value \\
\hline $\mathrm{P}_{\mathrm{n}}$ & $2.5 \mathrm{MW}$ \\
\hline $\mathrm{V}_{\mathrm{n}}$ & $575 \mathrm{~V}$ \\
\hline $\mathrm{p}$ & 2 \\
\hline $\mathrm{R}_{\mathrm{s}}$ & $6.3 \mathrm{~m} \Omega$ \\
\hline $\mathrm{L}_{\mathrm{d}}$ & $6.8 \mathrm{mH}$ \\
\hline $\mathrm{L}_{\mathrm{q}}$ & $1.49 \mathrm{~Wb}$ \\
\hline$\psi_{\mathrm{m}}$ & $10 \mathrm{Nm}$ \\
\hline $\mathrm{J}$ & $16 \mathrm{kNm}$ \\
\hline $\mathrm{T}_{\mathrm{m}}$ & \\
\hline \multicolumn{2}{|c|}{ DC Link } \\
\hline $\mathrm{C}_{\mathrm{dc}}$ & $60000 \mu f$ \\
\hline $\mathrm{V}_{\mathrm{dc}}$ & $4200 \mathrm{Vcc}$ \\
\hline $\mathrm{L}_{\mathrm{f}}$ & $1 \mathrm{mH}$ \\
\hline $\mathrm{f}_{\mathrm{r}}$ & $50 \mathrm{~Hz}$ \\
\hline $\mathrm{V}_{\mathrm{r}}$ & $470 \mathrm{~V}$ \\
\hline
\end{tabular}

\section{References}

1. Z. Zhang, Y. Zhao, W. Qiao, and L. Qu, IEEE Trans. Ind. Appl., 50, 4, 2331-2341, (2014).

2. BRASS CHILE S.A., "Proyecto Recuperacion de Energía Mineroducto Los Bronces - Las Tortolas," Santiago de Chile, 2005.

3. J.A.T. M. J. Sabatier, O. P. Agrawal, Advances in Fractional Calculus. Springer, 2007.

4. M.S. Tavazoei, IEEE Ind. Electron. Mag., 6, 3, 41-51, (2012).

5. M.Ö. Efe and S. Member, "Fractional Order Systems in Industrial Automation - A Survey," IEEE Trans. Ind. Informatics, vol. 7 , no. 4, pp. 582-591, 2011.

6. Y.Q. Chen, Proceedings ISRCS 2010, 58-63, (2010).

7. R. Melício, V.M.F. Mendes, J. P. S. Catalão, Energy Convers. Manag., 51, 6, 1250-1258, (2010).

8. R. Melício, V.M.F. M. F. Mendes, and J. P. S. P. S. Catalão, Proceedings 6th IET Int. Conf. PEMD 2012, P260-P260, (2012).

9. M. Seixas, R. Melício, and V.M.F. Mendes, Energy, 69, 357-369, (2014)

10. A. Kumar and K.T.B.P. Elizabeth, Proceedings Int. Conf. ICAGE 2014, 74-81, (2014).

11. A.J. Calderón, B.M. Vinagre, and V. Feliu, Signal Processing, 86, 10, 2803-2819, (2006).

12. S. Ghasemi, S. Member, and A. Tabesh, IEEE Trans. Energy Conversion, 29, 3, 780-787, (2014).

13. C. Wu and Y. Zhang, Proceedings of the $26^{\text {th }} 2014$ CCDC, 2064 2069, (2014)

14. H. Afghoul, F. Krim, D. Chikouche, A. Beddar, 3rd Int. Conf CEIT 2015, (2015).

15. R.E. Best, McGraw-Hill Education, (2003).

16. M.G. Daniel, A.M. Omar, T.O. Rubén, G.B. Abel, Procedia Technol., 7, 150-157, (2013).

17. B.M. Vinagre, A.C. Monje, Rev. Iberoam. Automática e Informática Ind., 3, 3, 5-23, (2006).

18. Y. Povstenko, Springer Book, 219, 211-225, (2015).

19. A.A. Kilbas, H.M. Srivastava, J.J. Trujillo, Elsevier Book, 129 204, (2006).

20. I. Podlubny, IEEE Trans. Automat. Contr., 44, 1, 208-214, (1999).

21. T. Pérez-Soriano, J. Fernández-Martínez, Master Thesis, Aalborg University, (2011).

22. D. Mehrzad, J. Luque, M. Capella-Cuenca, Master Thesis, Aalborg University, (2009).

23. A. Tepljakov, Master Thesis, Tallinn University of Technology (2011)

24. N. Phankong, S. Manmai, K. Bhumkittipich, and P. Nakawiwat, Energy Procedia, 34, 262-272, (2013).

25. A. Giannakis, A. Karlis, Y.L. Karnavas, Renewable Energy, 121, 489-501, (2018).

26. M. Rezkallah, A. Hamadi, A. Chandra, B. Singh, IEEE Trans. Ind. Elec., 65, 7, 5590-5600, (2018).

27. R.H. Park, Winter Conv. A.I.E.E., 352-354, (1929)

28. P. Mao, M. Zhang, and W. Zhang, J. Power Electron., 14, 5 , 1057-1068, (2014)

29. R. Teodorescu, M. Liserre, P. Rodríguez, John Wiley Book, (2011) 